\title{
Effect of Wood Waste on Acoustic and Magnetic Properties of Composite Bricks and Correlation between Sound Velocity and Magnetic Induction in These Bricks
}

\author{
Gilbert Ganga1,2,3*, Adolphe Kimbonguila Manounou ${ }^{1,3}$, Narcisse Malanda1,3, \\ Michel Dzondo Gadet ${ }^{1,2}$, Timothée Nsongo ${ }^{4,5}$
}

${ }^{1}$ National Advanced School of Engineering, University Marien Ngouabi, Brazzaville, Congo

${ }^{2}$ Center for Research and Initiation of Technology Projects (CRIPT), Brazzaville, Congo

${ }^{3}$ Laboratory of Mechanics, Energtics and Engineering, ENSP-UMNG, Brazzaville, Congo

${ }^{4}$ Facultyof Sciences and Technology, University Marien Ngouabi, Brazzaville, Congo

${ }^{5}$ Center of Géological and Mining Research, Brazzaville, Congo

Email: ^gangegilbert@gmail.com

How to cite this paper: Ganga, G., Manounou, A.K., Malanda, N., Gadet, M.D. and Nsongo, T. (2020) Effect of Wood Waste on Acoustic and Magnetic Properties of Composite Bricks and Correlation between Sound Velocity and Magnetic Induction in These Bricks. Open Journal of Composite Materials, 10, 15-25. https://doi.org/10.4236/ojcm.2020.101002

Received: October 8, 2019

Accepted: December 23, 2019

Published: December 26, 2019

Copyright $\odot 2020$ by author(s) and Scientific Research Publishing Inc. This work is licensed under the Creative Commons Attribution International License (CC BY 4.0).

http://creativecommons.org/licenses/by/4.0/ (c) (i) Open Access

\begin{abstract}
In the present study, kambala (botanical name: Chlorophora excelsa and Chlorophora regia) wood wastes were incorporated into stabilized earth bricks in order to test their acoustic insulation capacity of the walls; leading to better waste management from the timber industry. Two methods have been applied to determine the influence of the wood waste content in the stabilized earth bricks, on the weakening of the level of noise reception coming from the environmental medium, in an apartment built with composite bricks (earth + wood chips + cement). This influence has also been analyzed on the magnetic field induced by these bricks. The results showed that the level of sound reception through these bricks decreases with increasing wood waste content regardless of the method used (from $110 \mathrm{~dB}$ to $68 \mathrm{~dB}$, respectively for $\Phi_{b}=0 \%$ and $\left.\Phi_{b}=8 \%\right)$. The kambala wood waste in cement-stabilized clay bricks induces a magnetic field that increases with the wood waste content; the high contents of the wood chips causing an increase in the magnetic permeability of the composite medium. The correlation between the noise level and the magnetic field of the bricks shows that the noise level declines with increasing magnetic induction of the bricks.
\end{abstract}

\section{Keywords}

Kambala Tree, Wood Waste, Clay Brick, Sound Velocity, Magnetic Field 


\section{Introduction}

Congo is among the countries which produce wood in Central Africa. The processing of this wood by industries produces huge amounts of waste that raise a real management issue. However, wood is one of the materials commonly used in the building industry for acoustic and thermal insulation of compartments and music recording studios due to its ability to dampen, or even confine waves. Thanks to its cellular structure made of microscopic concavities arranged in array shown by Boustingorry, Brancheriau and Kellati [1] [2] [3], wood is most often used in its solid form to ensure proper insulation. These microscopic cavities serving as channels for the flow of sap through the tree, become cavities storing air in the case of a dry wood.

Today, several researchers as Khelifi, Taoukil and Tamba [4] [5] [6] showed in their work that wood can be used in the form of waste (chips and sawdust), incorporated at different contents in clay bricks $(C B)$. Most of these works are focused on the impact of wood waste $(W W)$ incorporated in earth blocks on mechanical, thermal and acoustic properties of $C B$ as shown by the work of: Ganga, Salif and Meukam [7] [8] [9] [10] [11]. The air stored by the wood waste embedded in the earth bricks, leads to an increase in the porosity of these composite bricks according to the content of wood waste.

The high noise level coming from an external source or from a neighbouring compartment is often a source of nuisance or even conflict between families living in apartments next to the same building. It can also be the source of certain diseases in some people; hence the need for insulating walls between two apartments.

As part of this work, we studied the ability of $W W$ incorporated in bricks to reduce the level of noise reception in a housing, using two (2) different methods.

The purpose of this work was to show that the wood used as waste in composite bricks can provide an acoustic barrier.

Wood waste induces a magnetic field in bricks. We also made a correlation between this magnetic field and the level of noise reception.

\section{Materials and Methods}

\subsection{Brick Making}

Bricks of dimensions: $16 \mathrm{~cm} \times 4 \mathrm{~cm} \times 4 \mathrm{~cm}$ were made, according to the proportions of Table 1 and materials below: a metal brick mold (see Figure 1(a)); a manual concrete press (see Figure 1(b)) with its accessories for compaction; a KERN KB-type electronic scale (range 10,100 g, $\mathrm{d}=0.1 \mathrm{~g}$ ).

The particle size of dry and untreated kambala wood chips (KWC) used in the manufacture of composite bricks ranges from 0.5 to $2 \mathrm{~mm}$ [5] [8].

The soil dried at $105^{\circ} \mathrm{C}$ and sieved with AFNOR 34 module sieve (diameter: 2 $\mathrm{mm})$ [12] was also used for making the bricks.

Twenty four (24) bricks per percentage of $W W$ were produced according to the following procedure: a sieved clay mass and cement corresponding to the 


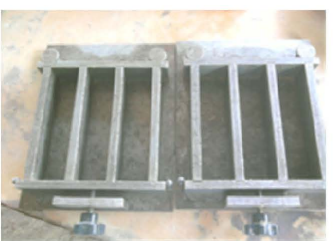

(a)

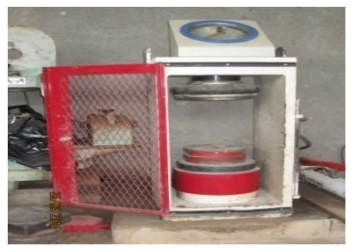

(b)

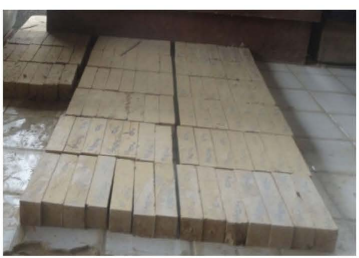

(c)

Figure 1. Materials for making bricks and presentation of a sample of clay bricks stabilized with $6 \%$ cement in which kambala chips are progressively incorporated. (a) Brick mould [9]; (b) Manual press with concrete; (c) Brick samples [9].

Table 1. Mixture proportions for bricks.

\begin{tabular}{|c|c|c|}
\hline $\mathrm{N}^{\circ}$ & Mixture proportions & Total \\
\hline 1 & $12,000 \mathrm{~g}(94 \%)$ ground $+720 \mathrm{~g}(6 \%)$ cement $+0 \% \mathrm{WW}$ & $12,720 \mathrm{~g}(100 \%)$ \\
\hline 2 & $12,000 \mathrm{~g}(92 \%)$ ground $+720 \mathrm{~g}(6 \%)$ cement $+240 \mathrm{~g}(2 \%) \mathrm{WW}$ & $12,960 \mathrm{~g}(100 \%)$ \\
\hline 3 & $12,000 \mathrm{~g}(90 \%)$ ground $+720 \mathrm{~g}(6 \%)$ cement $+480 \mathrm{~g}(4 \%) \mathrm{WW}$ & $13,200 \mathrm{~g}(100 \%)$ \\
\hline 4 & $12,000 \mathrm{~g}(88 \%)$ ground $+720 \mathrm{~g}(6 \%)$ cement $+720 \mathrm{~g}(6 \%) \mathrm{WW}$ & $13,440 \mathrm{~g}(100 \%)$ \\
\hline 5 & $12,000 \mathrm{~g}(86 \%)$ ground $+720 \mathrm{~g}(6 \%)$ cement $+960 \mathrm{~g}(8 \%) \mathrm{WW}$ & $13,680 \mathrm{~g}(100 \%)$ \\
\hline
\end{tabular}

formulation to be manufactured, was taken, weighed and poured into a tray; then knead with the trowel. Then, a quantity of $W W$ corresponding to the same formulation was taken, weighed, poured into the tank containing the preceding mixture (clay earth + cement). Then knead again until we obtain a homogeneous material. The mixture was gradually moistened with the mixing water, the percentage (22\%) of which was obtained by the Proctor Test according to standard NF-P 94-093 [13]; then kneads again. The molds (Figure 1(a)) were loaded; then worn on the press (Figure 1(b)). The compaction was carried out with a pressure force of $75 \mathrm{KN}$.

The bricks thus compacted are deposited on the bench of the laboratory (Figure $1(\mathrm{c})$ ) where they are dried up to 28 days at room temperature $\left(25^{\circ} \mathrm{C}\right.$ to $\left.27^{\circ} \mathrm{C}\right)[9]$.

\subsection{Measurement of Acoustic Properties}

Two experimental devices were used for sound measurement: the sound level meter (Figure 2(a)) and the Portable Ultrasonic Non-destructive Digital Indicating Tester (PUNDIT7) ultrasound device (Figure 2(b)). The measurements were taken at the physics laboratory of the "National Teacher Training College", away from any other sound source that could skew the results.

The length of the bricks was measured using a vernier calliper at 1/50th (0.02 $\mathrm{mm}$ accuracy). A Tesla-meter was used to measure the magnetic field induced by composite bricks.

We measured the sound of a radio transmitter placed $160 \mathrm{~mm}$ from the sound meter in the open air first; then, placed at the same distance (Figure 3(a)), in a hermetically sealed box, built with stabilized clay bricks $(S C B)$, mixed with $K W C$ 


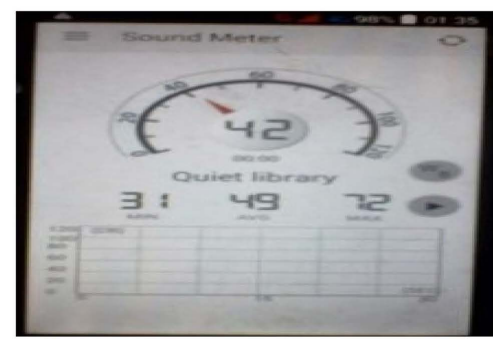

(a)

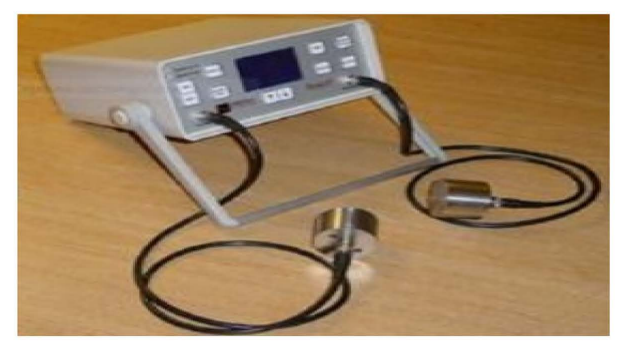

(b)

Figure 2. Materials for measuring the acoustic properties of bricks. (a) Sonometer; (b) Apparatus with ultrasound PUNDIT7 [9].

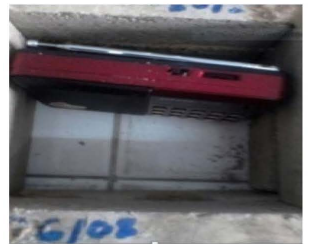

(a)

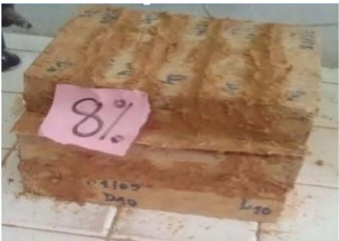

(b)

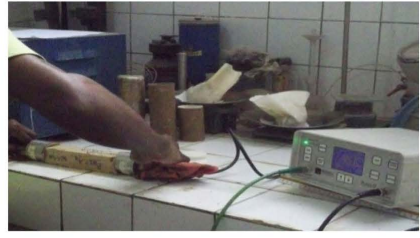

(c)

Figure 3. Measurement of the acoustic properties of bricks. (a) Radio transmitter between 4 walls; (b) Box in which the radio transmitter is fleeing; (c) Measurement of the propagation time of the wave.

at different levels, and with a clay mortar (Figure 3(b)) according to standard NF EN 61672-1 [14].

The speed being the quotient of the length of the specimen on the propagation time of the sound in the brick [8], we measured the time of travel of the wave in the brick placed between the two converters of the ultrasonic device PUNDIT7 (see Figure 3(c)) according to standard NF EN 12504-4 [15]. The measurements were carried out in the laboratory of the Office of Building Control and Public Works in the abbreviation "BCBTP" in Brazzaville.

\subsection{Magnetic Field Measurement}

Earth has a geomagnetic field due to the movement of molten rocks within the Earth's core; a field whose intensity varies according to where we are on the surface of the earth. The magnetic induction of cement-stabilized clay bricks mixed with $K W C$ at different grades was measured using a Tesla-meter. The measurement was done by placing the activated device on the brick according to standard NF EN 62233 [16].

\section{Results and Discussion}

\subsection{Presentation of the Results}

Consider a composite material clay + cement + wood. Let $\Phi_{(g+c)}$ be the mass proportion of the clay + cement mixture which constitutes the stabilized mixture (stabilized clay with $6 \%$ cement) and, $\Phi_{\mathrm{b}}$ the mass proportion of the wood chips $(W C)$ in the clay + cement + wood mixture. By definition, the mass proportion 
of $W C \Phi_{\mathrm{b}}$ in this composite is given by:

$$
\Phi_{b}=\frac{m_{b}}{m_{g+c}+m_{b}} \times 100
$$

where $m_{b}$ and $m_{(g+c)}$ are respectively the masses of $W C$ and the stabilized mixture clay + cement. In the same way, the mass proportion of the clay + cement mixture, noted $\Phi_{(g+c)}$ in the composite clay + cement + wood is given by:

$$
\Phi_{g+c}=\frac{m_{g+c}}{m_{g+c}+m_{b}} \times 100
$$

We study the influence of the $W C$ content on the acoustic properties of composite bricks (clays + cement + wood) according to the following composition (see Table 2).

To study the effect of adding $W C$ in the clay + cement mixture, the mixture B1 containing $0 \%$ wood chips is considered as a reference composite.

In order to allow an easy discussion of the results, the measured acoustic quantities (noise level, sound velocity and magnetic induction) in the composite containing $W C$ are standardized with respect to the reference acoustic quantities, measured in the medium containing $0 \%$ of $W C$.

Call $V_{0}, B_{0}$ and $L_{0}$, respectively the speed of sound, the magnetic induction and the noise level in the reference composite (clay + cement), and $V, B$ and $L$ respectively, the speed of sound, the magnetic induction and noise level in the composite with addition of $W C$. We define by $V^{*}, B^{*}$ and $L^{*}$ respectively the sound velocity, the magnetic induction and the noise level dimensionless (normed with respect to the acoustic quantities chosen as references) given by:

$$
\begin{aligned}
V^{*} & =\frac{V}{V_{0}} \\
B^{*} & =\frac{B}{B_{0}} \\
L^{*} & =\frac{L}{L_{0}}
\end{aligned}
$$

We discuss in the following, the effect of the addition of $W C$ on these adimensional quantities.

Table 2. Composite bricks in mass and proportion.

\begin{tabular}{cccccc}
\hline \multirow{2}{*}{$\begin{array}{c}\text { Studied } \\
\text { mixture }\end{array}$} & \multicolumn{2}{c}{ Mass composite mixture $(\mathrm{g})$} & \multicolumn{2}{c}{ Mixed mixture in proportion (\%) } \\
\cline { 2 - 6 } & $\begin{array}{c}\text { Clay + cement } \\
\left(m_{g+c}\right)\end{array}$ & $\begin{array}{c}\text { Wood chips } \\
\left(m_{b}\right)\end{array}$ & $\begin{array}{c}\text { Total } \\
\text { mass }\end{array}$ & $\begin{array}{c}\text { Proportion of clay } \\
+ \text { cement }\left(\Phi_{g+c}\right)\end{array}$ & $\begin{array}{c}\text { Proportion of } \\
\text { wood chips }\left(\Phi_{b}\right)\end{array}$ \\
\hline B1 & 12,720 & 0 & 12,720 & 100 & 0 \\
B2 & 12,720 & 240 & 12,960 & 98 & 2 \\
B3 & 12,720 & 480 & 13,200 & 96 & 4 \\
B4 & 12,720 & 720 & 13,440 & 94 & 6 \\
B5 & 12,720 & 960 & 13,680 & 92 & 8 \\
\hline
\end{tabular}




\subsection{Influence of Wood Chip Content on the Acoustic Properties of Clay Bricks Stabilized at 6\% Cement}

Table 3 summarizes the attenuation values of acoustic properties of $C B$ stabilized at $6 \%$ cement as a function of $K W C$ content. We took $110 \mathrm{~dB}$ reference noise level and $2194 \mathrm{~m} / \mathrm{s}$ reference sound speed for the brick without adding KWC.

The sound velocity measured in the reference brick B1 (without the addition of $K W C$ ) is $2194 \mathrm{~m} / \mathrm{s}$, which is about 6 times greater than the rate of propagation of sound in the air. The addition of $8 \%$ of $K W C$ in the reference composite B1 results in a reduction of this ratio to 4.5 (i.e. $1560 \mathrm{~m} / \mathrm{s}$ for brick B5).

Figure 4 shows the variation of the adimensional speed $V^{*}$ of the sound as a function of the $W C$ content $\Phi_{b}$. A linear decrease of the speed of sound is observed with the increase in $K W C$ content.

This linear decrease in the average sound velocity as a function of the increase in wood fiber content by ultrasonic testing was also reported by Mekhermeche [17] in the conclusions of his study on composite bricks with date palm fibers.

This linear decay is described by the mathematical model (8):

$$
V^{*}=V_{0}^{*}-I_{a} \Phi_{b}
$$

where $V_{0}^{*}$ is the adimensional speed of sound measured in the reference brick (clay + cement) without adding $K W C\left(\Phi_{b}=0 \%\right)$ and $I_{a}=0.036$ is the attenuation coefficient of sound propagation in the brick following the addition of $K W C$. Recall that $\Phi_{b}$ is the $K W C$ addition content defined by Equation (1). The value of the attenuation coefficient $I_{a}$ is the result of a linear adjustment of the experimental data made with the Gnuplot software via the Marquardt-Levenberg method.

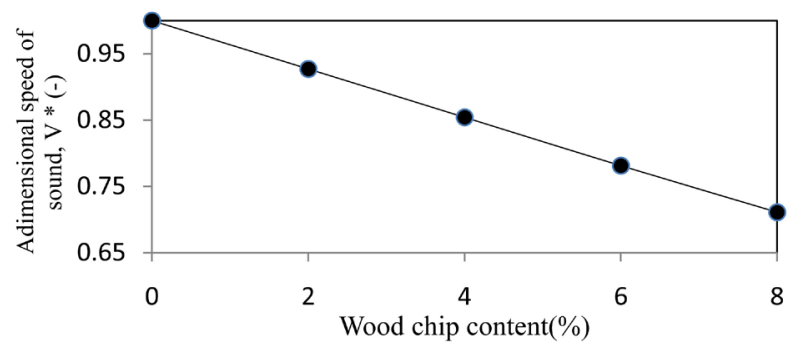

Figure 4. Variation in adimensional velocity of sound propagation as a function of KWC content in clay bricks stabilized at $6 \%$ cement.

Table 3. Attenuation values of the acoustic properties of clay bricks stabilized at $6 \%$ cement by adding kambala chips.

\begin{tabular}{cccc}
\hline Bricks & $\begin{array}{c}\text { Kambala wood } \\
\text { chips content (\%) }\end{array}$ & $\begin{array}{c}\text { Attenuation of } \\
\text { the noise level \% }\end{array}$ & $\begin{array}{c}\text { Attenuation of the } \\
\text { speed of sound (\%) }\end{array}$ \\
\hline B2 & 2 & 36 & 8 \\
B3 & 4 & 40 & 17 \\
B4 & 6 & 53 & 28 \\
B5 & 8 & 61 & 41 \\
\hline
\end{tabular}


We can define from the relation (7), an optimal content of $K W C \Phi_{b o p t}$, which would lead to a speed of propagation of zero sound in the brick.

$$
\Phi_{b o p t}=\frac{V_{0}^{*}}{I_{a}}
$$

This optimum content of $K W C$ that gives a perfect acoustic insulation is estimated at about $27.56 \%$ according to the relation (7) resulting from the predictive model (6). According to this theoretical model, a $K W C$ content of about $23 \%$ would make a composite brick that would give a speed of sound propagation close to that of air.

Figure 5 shows the variation in the noise level generated by a transmitter source, as a function of the $K W C$ proportion in the $C B$ stabilized at $6 \%$ cement.

This curve shows that this level decreases rapidly, then more and more slowly as the $K W C$ content increases. The addition of $2 \% K W C$ results in an attenuation of approximately $36 \%$ of the noise level whereas this attenuation does not exceed $10 \%$ when the $K W C$ content varies from $2 \%$ to $8 \%$ (Table 3 ). The addition of $K W C$ in the reference brick (clay + cement) leads to an acoustic insulation that can reduce the noise level up to $62 \%$ for the proportions studied (from $110 \mathrm{~dB}$ to $68 \mathrm{~dB}$, respectively for $\Phi_{b}=0 \%$ and $\Phi_{b}=8 \%$ ).

The decay of the noise level with the increase in $K W C$ content observed in Figure 5 can be described by the model (8).

$$
L^{*}=0.909 \mathrm{e}^{-0.05 \Phi_{b}}
$$

The sound attenuation observed in Figure 4 following the addition of $K W C$ in the composite brick (clay + cement), could be explained by an increase in the porosity of the medium, induced by the alveolar structure of the wood consisting of microscopic cavities organized in a network that would trap air [1].

This sound attenuation was also observed by Cérézo [18] who showed in the conclusions of his thesis work from the Kundt tube test that the absorption coefficient of the sound wave increases with the increase in hemp fiber content.

In the rest of the paper, we propose a correlation between the evolution of the acoustic properties and the density of $C B$ stabilized at $6 \%$ cement.

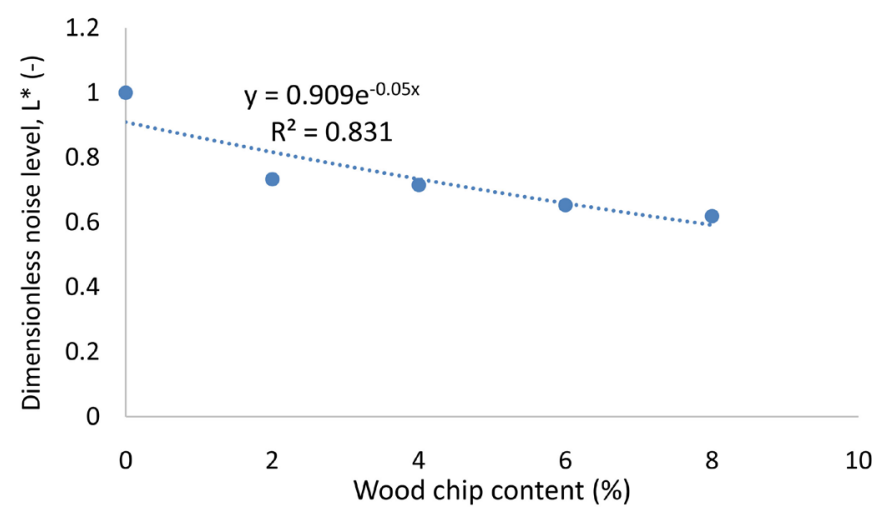

Figure 5. Variation of the level of noise reception as a function of KWC content in CB stabilized at $6 \%$ cement. 


\subsection{Influence of the Wood Chips Content on the Magnetic Induction of Stabilized Clay Bricks at $6 \%$ of Cement Content}

Figure 6 shows the influence of $K W C$ content on magnetic induction. Magnetic induction increases with the addition of $K W C$. However, a $6 \%$ addition of $K W C$ results in only $1 \%$ increase in magnetic induction. For these contents, the incorporation of the $K W C$ in the reference brick does not seem to significantly disturb the magnetic field of the medium. However, at $8 \%$ of $K W C$, the magnetic field disturbance reaches $5.56 \%$ compared to the magnetic field of the reference brick. The increased increase in magnetic induction could be explained by an increase in the porosity of the brick, induced by the alveolar structure of the introduced wood, consisting of microscopic cavities organized in a network that would trap air. The increase in pores, created by the addition of wood, leads to an increase in the magnetic permeability, and therefore in the magnetic induction.

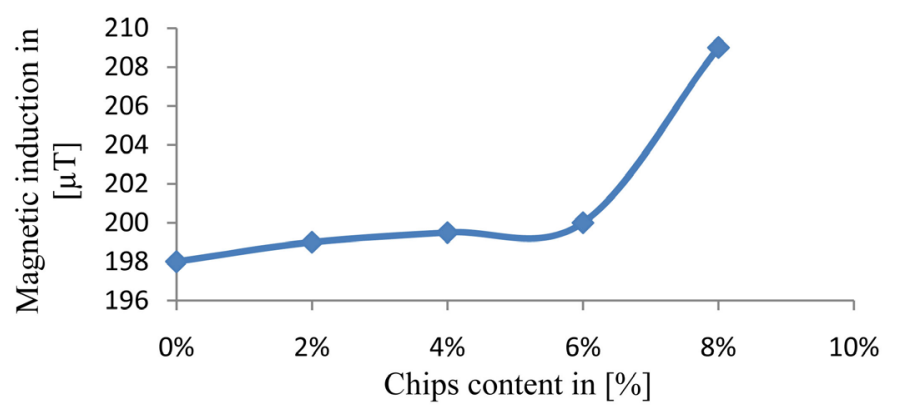

Figure 6. Change in the magnetic induction of stabilized clay bricks at $6 \%$ of cement content depending on the wood chips content.

\subsection{Relationship between the Acoustic Properties of Stabilized Clay Bricks and Their Density}

Figure 7 shows the speed of sound propagation and noise level dimensionless as a function of the density of the bricks.

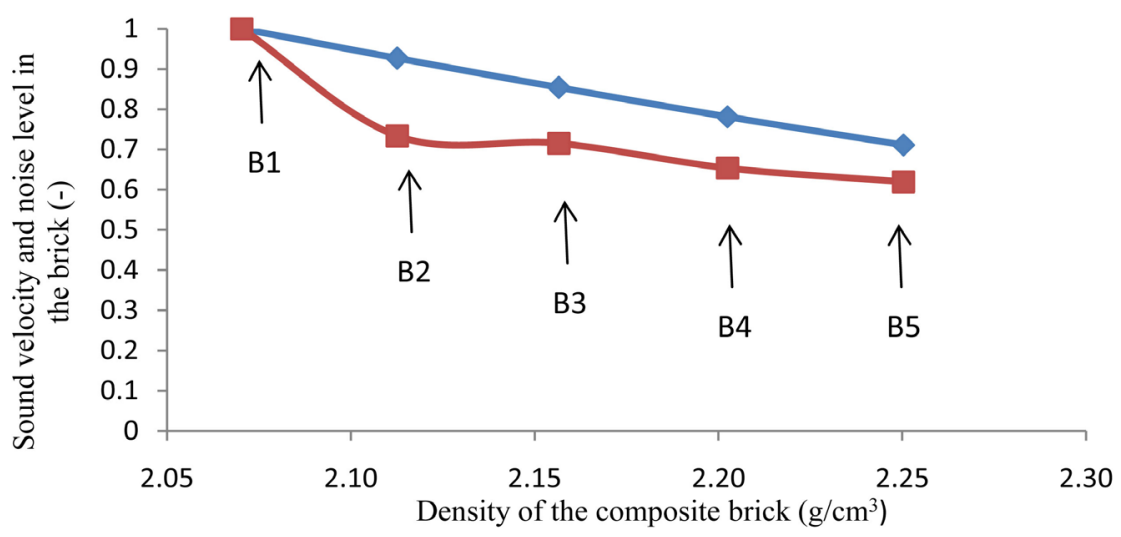

Figure 7. Relationship between the density variation and the acoustic quantities of $\mathrm{CB}$ stabilized at $6 \%$ cement and containing KWC. The red curve represents the sound velocity; the blue curve represents the noise level. 
This figure shows that the speed of sound propagation and the noise level are inversely proportional to the density of the bricks. The increase in the density of the bricks with the $K W C$ content leads to an attenuation of the propagation speed of the sound and the noise level. The speed of sound propagation in bricks is well correlated with the density of bricks by the power law (9).

$$
V^{*}=\left[\frac{\rho\left(\Phi_{b}\right)}{\rho_{0}}\right]^{-\alpha}
$$

With $\rho_{0}=2.07 \mathrm{~g} / \mathrm{cm}^{3}$ is the density of the composite brick B1 (without addition of $K W C$ ) and $\alpha=4 \pm 0.05$ is the slope of the power law which reflects the attenuation rate sound propagation in bricks.

\subsection{Relationship between the Noise Level and the Magnetic Induction of Stabilized Clay Bricks}

Figure 8 shows that there is a correlation between magnetic induction and the level of noise reception through $S C B$, incorporating $K W C$. In fact, the level of noise reception decreases with the increase in the magnetic field of the bricks for $W C$ contents of less than $6 \%$, whereas it seems to reach an asymptotic regime at high levels.

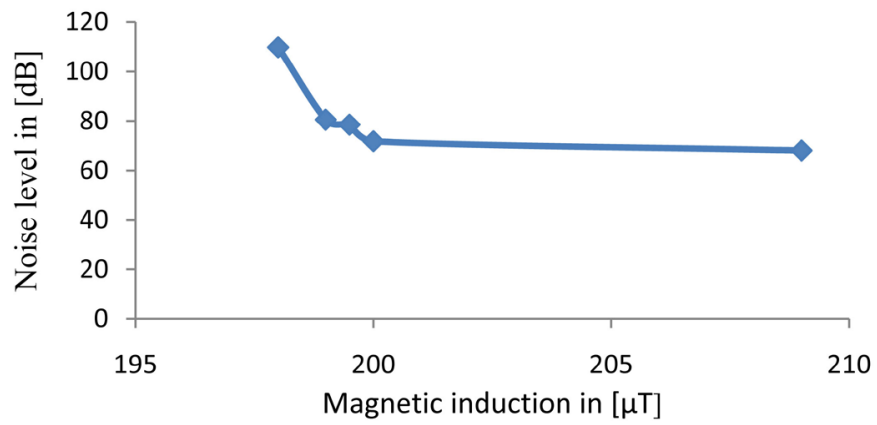

Figure 8. Relationship between the noise level and the magnetic induction of CB stabilized at $6 \%$ of cement and with WC.

\section{Conclusions and Perspectives}

In this paper, the influence of incorporating $K W C$ into cement- $S C B$ on acoustic and magnetic properties has been studied experimentally.

We have shown that the addition of $K W C$ in cement- $S C B$ has a significant influence on the acoustic properties of these bricks. Indeed, it leads to an attenuation of the level of noise reception and the speed of sound propagation, which constitutes an interesting alternative for the acoustic insulation of buildings and could also constitute an economic solution. Two mathematical models have been proposed to satisfactorily describe the decrease in noise level and sound propagation velocity in bricks as a function of $K W C$ content.

The effect of adding $K W C$ in $C B$ on the magnetic properties remains to be specified. However, we have shown that at low levels (up to 6\%), the addition of $K W C$ has little influence on the magnetic induction compared to the reference 
brick that does not contain $W C$. This influence tends to become significant at $8 \%$ of $W C$.

Several correlations have been obtained between the acoustic, magnetic and physical properties of composite bricks. Indeed, the speed of sound propagation decreases in power law with the increase of the density of the bricks. The level of noise reception in the bricks decreases with the increase of the magnetic induction until reaching an asymptotic regime.

The effect of the addition of $K W C$ on the magnetic properties will be the subject of further study, especially at high levels.

\section{Acknowledgements}

We do thank the Managing Director of the "Office of Building Control and Public Works", and the physics head of department of "National Teacher Training College", who have enabled and allowed us to carry out some experimental tests in their laboratory.

\section{Conflicts of Interest}

The authors declare no conflicts of interest.

\section{References}

[1] Boustingorry, P. (2002) Elaboration of a Gypsum Matrix Composite Material and Fragmented Wood Reinforcement: Improvement of the Resistance to Screwing of Prefabricated Gypsum Products. Doctoral Thesis, ENSM de St-Etienne, France.

[2] Brancheriau, L. (2013) Acoustic and Ultrasonic Characterization of the Products Wood and Composites. Accreditation to Supervise Research (HDR); Université Montpellier 2, France.

[3] Kellati, N., El Bouardi, A., Ajzoul, T. and Ezbakhe, H. (2007) Study of Thermophysical and Acoustic Properties of Compact and Granular Cork (in French). Review of Renewable Energies 'CER'07, Oujda, 241-244.

[4] Khelifi, W. and Belouettar, R. (2012) Influence of the Nature of the Shavings on the Physical and Mechanical Characteristics of the Concretes Containing Shavings. 20th University Meeting of Civil Engineering, Chambéry, France.

[5] Tamba, S., Voumbo, M.L, Wareme, A., Gaye, S. and Sissoko, G. (2007) Durability of Lightweight Concrete Made of Wood Chips. Journal of Science, 7, 67-72.

[6] Taoukil, D., El bouardi, A., Ajzoul, T. and Ezbakhe, H. (2011) Mechanical and Water Characterization of a Lighter Concrete with Wood Waste. International Journal of Solar Technology, 43, 1-7.

[7] Ganga, G., Nsongo, T., Elenga, H., Moukala, L.M., Nzabana, A. and Ngoro, E.F. (2017) Impact of the Wood Chips Content on the Mechanical Properties and Relation between the Young Modulus and the Compressive Strength of Clay Bricks Stabilized. Research Journal of Physical Sciences, 5, 1-5.

[8] Ganga, G. (2016) Study of the Influence of the Content of the Chips of Wood on the Mineralogical Structure, Mechanical and Acoustic Properties of Laterite and Clay Bricks. Marien Ngouabi University, Brazzaville, Congo.

[9] Ganga, G., Nsongo, T., Elenga, H., Mabiala, B., Tamo, T.T. and Nzonzolo (2014) Effect of Incorporation of Chips and Wood Dust Mahogany on Mechanical and 
Acoustic Behavior of Brick Clay. Journal of Building Construction and Planning Research, 2, 198-208. https://doi.org/10.4236/jbcpr.2014.23018

[10] Gaye, S. (2001) Characterization of the Mechanical Acoustic and Thermic Properties, of Local Materials of Construction in Senegal. Doctorate of Science State, University Cheikh Anta Diop, Senegal.

[11] Meukam, P. (2004) Characterization of Local Materials for the Thermal Isolation of the Buildings. Ph.D. Thesis, University of Yaoundé I, Cameroon.

[12] NF EN 933-2(1996) Test to Determine the Characteristics of the Aggregates-Part 2: Determination of the Granularity-Sieve of Control-Nominal Dimensions of the Openings. French Standard Association, France.

[13] NF-P 94-093 (1999) Grounds: Recognition and Tests-Determination of the References of Compaction of a Material-Normal Proctor Test-Modified Proctor Test. French Standard Association, France.

[14] NF EN 61672-1 (2014) Electrotechnics-Sound Meter-Part 1: Specifications. French Standard Association, France.

[15] NF EN 12504-4 (2005) Tests for Concrete in the Structures-Part 2: Non-Destructive Tests-Determination of the Propagation Velocity of the Sound. French Standard Association, France.

[16] NF EN 62233 (2013) Methods of Measurement of the Magnetic Fields of the Apparatuses Electro Domestic and Similar in Relation to the Human Exposure. French Standard Association, France.

[17] Mekhermeche, A. (2012) Contribution à l'etude des propriétés mécaniques et thermiques des briques en terre en vue de leur utilisation dans la restauration des Ksours sahariennes. Mémoire de Magister, Universite Kasdi Merbah, Ouargla, Algérie.

[18] Cerezo, V. (2005) Propriétés mécaniques, thermiques et acoustiques d'un matériau à base de particules végétales: Approche expérimentale et modélisation théorique. Thèse de doctorat, Institut National des Sciences Appliquées de Lyon, Lyon.

\section{Abbreviations}

WW: Wood Waste

CB: Clay Bricks

KWC: Kambala Wood Chips

PUNDIT7: Portable Ultrasonic Non-Destructive Digital Indicating Tester

WC: Wood Chips 